\title{
ISOLATION OF ACTINOMYCES PYOGENES FROM FISTULA IN AN ELEPHANT
}

\author{
B.S.M. Ronald ${ }^{1}$, K. Sukumar, M.S. Meenachiselvan and \\ N. Dorairajan \\ ${ }^{1}$ Assistant Professor \\ Department of Microbiology, Veterinary College and Research \\ Institute, Namakkal, Tamil Nadu 637001, India.
}

An elephant was admitted with abscess and fistula behind the right ear lobe following an accident, to the Veterinary College and Research Institute, Namakkal. The pachyderm was previously treated by a local veterinarian for two weeks without much improvement. Pus material was collected to identify the organism and for antibiotic sensitivity test. The sample was inoculated in nutrient broth and Roberston's cooked meat medium (Himedia) and further streaked on to various selective media like MacConkey agar, Tryptose soy agar, Potassium tellurite agar, Mannitol salt agar, Sabouraud's dextrose agar, and Cetrimide agar and incubated at $37^{\circ} \mathrm{C}$. It was also streaked on anaerobic egg agar (Himedia) and kept in an anaerobic jar with $\mathrm{Hi}$ indicator and Hi Gas Pack (Himedia) and incubated at $37^{\circ} \mathrm{C}$.

Growth on the medium were further subcultured to isolate pure colonies and biochemical tests were done to identify up to species level following Bergey's manual of determinative bacteriology (Breed et al., 1974).

The culture revealed the presence of Actinomyces pyogenes, Pseudomonas aeruginosa, Micrococcus luteus, Klebsiella orytoca, Proteus mirabilis, Staphylococcus aureus and Corynebacterium ulcerans. Antibiotics sensitivity test was conducted for each organism as per Bauer et al. (1966) (Table).

All the organisms isolated were sensitive to Enrofloxacin and Amoxycillin and were resistant to Penicillin, Neomycin and Streptomycin. Actinomyces pyogenes, Pseudomonas aeruginosa, Staphylococcus aureus and Corynebacterium ulcerans could be involved in producing abscess and fistula since they are capable of producing pyogenic infections whereas Klebsiella oxytoca, Proteus mirabilis and Micrococcus luteus may be clinical contaminants from the skin of the animal. The elephant was treated with combination of Enrofloxaclin and Amoxycillin along with other supportive therapy. A marked improvement was noticed and the animal was discharged after one month of treatment.

\section{References}

Bauer, A.W., W.M. Kirby, J.C. Sherris and M. Truck (1966). Antibiotic susceptibility testing by a standard single disc method. Am. J. Clin. Path., 45: 493-496.

Breed, R.S., E.G.D. Murray and N.R. Smith (1974). Bergey's Manual of Determinative Bacteriology 8th Edition. Williams and Wilkins, Baltimore.

\begin{tabular}{|c|c|c|c|c|c|c|c|c|c|c|}
\hline \multirow[t]{2}{*}{ Name of the organism } & \multirow[b]{2}{*}{ Co } & \multirow[b]{2}{*}{ C } & \multirow[b]{2}{*}{ En } & \multicolumn{3}{|c|}{ Antibiotic discs. } & \multirow[b]{2}{*}{ G } & \multirow[b]{2}{*}{$\mathrm{Am}$} & \multirow[b]{2}{*}{ Ak } & \multirow[b]{2}{*}{$\mathrm{N}$} \\
\hline & & & & $\mathrm{Ci}$ & St & $\mathbf{P}$ & & & & \\
\hline 1. Actinomyces pyogenes & $\mathrm{R}$ & $\mathrm{R}$ & S & I & $\mathrm{R}$ & $\mathrm{R}$ & $\mathrm{R}$ & $\mathrm{S}$ & $\mathrm{R}$ & $\mathrm{R}$ \\
\hline 2. Pseudomonas aeruginosa & $\mathrm{R}$ & S & $\mathrm{S}$ & 1 & $\mathrm{R}$ & $\mathrm{R}$ & $\mathrm{S}$ & $\mathrm{S}$ & $\mathrm{S}$ & $\mathrm{R}$ \\
\hline 3. Klebsiella oxytoca & I & S & $\mathrm{S}$ & $\mathrm{S}$ & $\mathrm{R}$ & $\mathrm{R}$ & $\mathrm{S}$ & $S$ & S & $\mathrm{R}$ \\
\hline 4. Proteus mirabilis & $\mathrm{S}$ & $S$ & S & $\mathrm{S}$ & $\mathrm{S}$ & $\mathrm{R}$ & $\mathrm{S}$ & S & S & I \\
\hline 5. Micrococcus luteus & $\mathrm{S}$ & S & $\mathrm{S}$ & S & $\mathrm{R}$ & I & $\mathrm{R}$ & S & I & I \\
\hline 6. Staphylococcus aureus & I & I & $\mathrm{S}$ & $\mathrm{R}$ & $\mathrm{R}$ & I & $\mathrm{R}$ & $\mathrm{S}$ & $\mathrm{R}$ & $\mathrm{R}$ \\
\hline 7. Corynebacterium ulcerans & $\mathrm{R}$ & $\mathrm{R}$ & $S$ & $\mathrm{R}$ & $\mathrm{R}$ & $\mathrm{R}$ & $\mathrm{R}$ & $S$ & $\mathrm{R}$ & $\mathrm{R}$ \\
\hline $\begin{array}{l}\text { Co - Cotrimoxazole } \\
\text { C - Chloramphenicol } \\
\text { En - Enrofloxacin } \\
\text { Ci - Ciprofloxacin }\end{array}$ & $\begin{array}{l}\text { St }- \\
P-P \\
\text { G-C } \\
\text { Am }\end{array}$ & $\begin{array}{l}\text { G-Gentamicin } \\
\text { Am - Amoxycillin }\end{array}$ & & & & \multicolumn{2}{|c|}{$\mathrm{N}$ - Neromycin } & \multicolumn{2}{|c|}{ Himedia } & \\
\hline S - Sensitive & \multicolumn{3}{|c|}{ R-Resistant } & & & \multicolumn{2}{|c|}{ I-Intermediate. } & & & \\
\hline
\end{tabular}

